SCHWEIZERISCHE ZEITSCHRIFT FUR HYDROLOGIE BIRKHÄUSER VERLAG BASEL

Vol. XIX 1957 Facs. 1

\title{
Die Grundlagen
}

\section{der. Fällungs-, Enthärtungs- und Entsalzungs- verfahren in der Wasseraufbereitung}

Von Dr. F. ZEHENDER, EAWAG, Zürich 
Das Ziel der Wasseraufbereitung ist es, im Hinblick auf einen bestimmten Verwendungszweck die Wasserzusammensetzung zu ändern. Ungelöste Stoffe werden mittels mechanischer Verfahren, zum Beispiel durch Sandfiltration, entfernt, während zur Beseitigung von echt oder kolloidal gelösten Verbindungen vorwiegend chemische Verfahren Anwendung finden. Im folgenden soll auf die Grundlagen einiger chemischer Aufbereitungsverfahren eingetreten werden.

\section{Enthärtung durch Fällung}

In vielen, in der Natur vorkommenden Wässern sind hauptsächlich die Salze des Kalziums und des Magnesiums enthalten. Sie werden kurz als «Härte» bezeichnet. Man unterscheidet zwischen der Karbonathärte oder der vorübergehenden Härte einerseits und der permanenten oder bleibenden Härte anderseits. Von vorübergehender Härte wird gesprochen, wenn Bikarbonate vorliegen. Sie zersetzen sich beim Erhitzen des Wassers, wobei die Härte zum grössten Teil ausfällt. Von bleibender Härte spricht man beim Vorliegen der Sulfate, Chloride oder Nitrate des Kalziums oder Magnesiums. Diese Salze fallen beim Kochen des Wassers nicht aus.

Die als «Härte» bezeichneten, im Wasser enthaltenen Stoffe sind aus verschiedenen Gründen unerwünscht. Einerseits bilden sie beim Waschen in Berührung mit Seife die Kalkseifen, die als Niederschlag auf der Wäsche einen «harten» Belag hinterlassen. Anderseits bilden sich mehr oder weniger "harte» Ablagerungen in Heisswasserapparaten, das heisst in Boilern, Dampfkesseln sowie in Leitungssystemen. Namentlich zur Verhinderung der Kesselsteinbildung muss das Brauchwasser im allgemeinen 
aufbereitet werden. Im übrigen sei erwähnt, dass man beim Waschen mit hartem Wasser mehr Seife benötigt als bei der Verwendung von weichem Wasser und dass in der Textilindustrie weiches Wasser bevorzugt wird, weil es der Ware einen « weichen Griff» zu geben vermag.

Wir wollen nun prüfen, welche Bestandteile des Wassers zu harten Belägen in Leitungssystemen und Heisswasserapparaten führen, welche Verbindungen sich dabei ablagern und wie die Ablagerungen zustande kommen. Um diese Fragen zu diskutieren, seien die hauptsächlichsten Ionen und Moleküle, die in einem Rohwasser vorkommen können, zusammengestellt. Es sind dies die Kationen $\mathrm{Ca}^{++}, \mathrm{Mg}^{++}, \mathrm{Na}^{+}, \mathrm{K}^{+}, \mathrm{H}^{+}$, die Anionen $\mathrm{HCO}_{3}^{-}, \mathrm{CO}_{3}^{--}, \mathrm{Cl}^{-}, \mathrm{NO}_{3}^{-}, \mathrm{SO}_{4}^{--}, \mathrm{OH}^{-}$sowie Kohlensäure und Kieselsäure. Von diesen teils ionisierten, teils nicht ionisierten Teilchen fallen folgende zur Bildung schwerlöslicher Verbindungen von vorneherein ausser Betracht, nämlich Natrium, Kalium, Nitrat und Chlorid. Sie müssen somit nicht weiter berücksichtigt werden.

Nun wenden wir uns den in der Regel vorherrschenden Ionen zu, dem Kalzium-, dem Magnesium- und dem Bikarbonation. Wir betrachten zuerst das Kalziumbikarbonat und stellen fest, dass die Löslichkeit dieser Verbindung recht beträchtlich sein kann. In der Natur sind Härtewerte von 30 frz. Graden keine Seltenheit. Im Experiment kann man noch viel höhere Konzentrationen erzielen, doch muss wegen der massgebenden Gleichgewichtsbedingungen mit zunehmendem Gehalt an Kalziumbikarbonat das $\mathrm{pH}$ sinken bzw. die Konzentration an freier Kohlensäure steigen. Der Gleichgewichtszustand kann wie folgt formuliert werden:

$$
\mathrm{Ca}\left(\mathrm{HCO}_{3}\right)_{2} \rightleftarrows \mathrm{CaCO}_{3}+\mathrm{H}_{2} \mathrm{CO}_{3}
$$

Wird das Gleichgewicht gestört, zum Beispiel im Sinne der Gleichung von links nach rechts, sei es durch Erhitzen der Lösung (wobei Kohlensäure entweicht), sei es durch Zugabe von Lauge, so fällt Kalziumkarbonat aus. Die Gleichgewichtsverhältnisse werden durch drei Konstanten beherrscht, nämlich durch das Löslichkeitsprodukt des Kalziumkarbonats

$$
\begin{gathered}
L=\left(\mathrm{Ca}^{+}\right) \cdot\left(\mathrm{CO}_{3}^{--}\right)=4,55 \cdot 10^{-8} \text { bei } 25^{\circ} \mathrm{C}, \\
\text { vgl. LARSON und BUSWELL } \left.[1]^{1}\right),
\end{gathered}
$$

die erste Dissoziationskonstante der Kohlensäure

$$
K_{1}=\frac{\left(\mathrm{H}^{+}\right) \cdot\left(\mathrm{HCO}_{3}^{-}\right)}{\left(\mathrm{H}_{2} \mathrm{CO}_{3}\right)}=4,45 \cdot 10^{-7} \text { bei } 25^{\circ} \mathrm{C},
$$

1) Die Ziffern in eckigen Klammern verweisen auf das Literaturverzeichnis, Seite 101. 
und die zweite Dissoziationskonstante der Kohlensäure

$$
\begin{gathered}
K_{2}=\frac{\left(\mathrm{H}^{+}\right) \cdot\left(\mathrm{CO}_{3}^{-}-\right)}{\left(\mathrm{HCO}_{3}^{-}\right)}=4,69 \cdot 10^{-11} \text { bei } 25^{\circ} \mathrm{C}, \\
\text { vgl. HARNED und Scholes [3]. }
\end{gathered}
$$

Wie aus (2) hervorgeht, ist die Löslichkeit des Kalziumkarbonats gering. Sie wird von E. Nordell [4] für $100^{\circ} \mathrm{C}$ mit $\mathrm{I}_{3} \mathrm{mg} \mathrm{CaCO}_{3} / 1$ angegeben (vgl. Tabelle). Beim Erhitzen eines Wassers, das Kalziumbikarbonat enthält, fällt also so viel Kalziumkarbonat aus, dass die verbleibende Härte nur noch $I, 3$ frz. Grade beträgt.

Löslicbkeiten pon Kalzium- bzw. Magnesiumkarbonat oder -bydroxyd bei $100^{\circ} \mathrm{C}$,

\begin{tabular}{|c|c|c|}
\hline & Karbonat & Hydroxyd \\
\hline $\begin{array}{l}\text { Kalzium- } \\
\text { Magnesium- }\end{array}$ & $\begin{array}{l}13 \\
75\end{array}$ & $\begin{array}{r}888 \\
8\end{array}$ \\
\hline
\end{tabular}
angegeben als $\mathrm{mg} \mathrm{CaCO}_{3} / \mathrm{l}$ (nach E. NoRdedu [4])

Die Löslichkeit von Magnesiumkarbonat ist erheblich grösser als diejenige von Kalziumkarbonat. Sie beträgt $75 \mathrm{mg} / \mathrm{l}\left(\right.$ als $\mathrm{CaCO}_{3}$ bei $\mathrm{IO0}^{\circ}$ ). Beim Vorliegen von Kalzium- und Magnesiumbikarbonat wird somit zuerst das schwerlösliche Kalziumkarbonat ausfallen. Es ist aber noch zu berücksichtigen, dass in der Hitze das Magnesiumkarbonat zerfällt, wobei sich unter Abgabe von Kohlensäure das schwerlösliche Magnesiumhydroxyd bildet:

$$
\mathrm{Mg}\left(\mathrm{HCO}_{3}\right)_{2}+2 \mathrm{H}_{2} \mathrm{O}=\mathrm{Mg}(\mathrm{OH})_{2}+2 \mathrm{H}_{2} \mathrm{CO}_{3} .
$$

Gemäss Tabelle ist das Kalziumhydroxyd 8oomal leichter löslich als das Magnesiumhydroxyd. Fällt man nicht durch Erhitzen der Lösung, sondern durch Zugabe von Lauge oder Soda, so wird auch in diesem Falle das Kalzium als Karbonat, das Magnesium dagegen als Hydroxyd ausgeschieden, als Folge der in der Tabelle angegebenen Löslichkeiten [vgl. Gleichung (8)].

Eine weitere schwerlösliche Substanz ist das Kalziumsulfat, dessen Löslichkeit bei $40^{\circ} \mathrm{C}$ ein Maximum aufweist und bei dieser Temperatur noch ziemlich hoch ist. Oberhalb von $100^{\circ} \mathrm{C}$ sinkt die Löslichkeit jedoch beträchtlich, weshalb namentlich in Dampfkesseln für hohe Drucke Gips- 
ablagerungen vorkommen können. Schliesslich sei noch auf die Kieselsäure hingewiesen, welche sich als solche, als Kalziumsilikat oder auch als Aluminosilikat ablagern kann.

Beim Kalkbydratperfabren (auch «Entkarbonisierung» genannt) wird von den beschriebenen Löslichkeits- und Gleichgewichtsverhältnissen Gebrauch gemacht. Das Verfahren beruht darauf, dass bei Zugabe von Kalziumhydroxyd zu einem kalziumbikarbonathaltigen Wasser eine pH-Verschiebung nach der alkalischen Seite eintritt, worauf dann Kalziumkarbonat gemäss folgender Gleichung ausfällt:

$$
\mathrm{Ca}\left(\mathrm{HCO}_{3}\right)_{2}+\mathrm{Ca}(\mathrm{OH})_{2}=2 \mathrm{CaCO}_{3}+2 \mathrm{H}_{2} \mathrm{O} \text {. }
$$

Der entstandene Niederschlag kann durch Absetzen oder Filtration vom Wasser abgetrennt werden. In neuerer Zeit ist das Verfahren in der Weise modifiziert worden, dass durch Erzeugung einer turbulenten Strömung bestimmter Stärke im Reaktionsgefäss die Fällung des Kalziumkarbonats beschleunigt wird, wobei sich der Niederschlag nicht in Form eines feinen Schlammes, sondern als kleine Körner abscheidet (Spiractor-, Wirbosverfahren).

Eine weitere Anmendung ist das Kalk-Soda-Verfabren, bei welchem die Kalziumverbindungen in $\mathrm{CaCO}_{3}$ und diejenigen des Magnesiums in $\mathrm{Mg}(\mathrm{OH})_{2}$ übergeführt und hierauf vom Wasser abgetrennt werden. Die Reaktionsgleichungen lauten:

a) Beim Vorliegen von Kalziumsulfat:

$$
\mathrm{CaSO}_{4}+\mathrm{Na}_{2} \mathrm{CO}_{3}=\mathrm{CaCO}_{3}+\mathrm{Na}_{2} \mathrm{SO}_{4} \text {. }
$$

b) Beim Vorliegen von Magnesiumbikarbonat:

$$
\mathrm{Mg}\left(\mathrm{HCO}_{3}\right)_{2}+2 \mathrm{Ca}(\mathrm{OH})_{2}=2 \mathrm{CaCO}_{3}+\mathrm{Mg}(\mathrm{OH})_{2}+2 \mathrm{H}_{2} \mathrm{O} .
$$

c) Beim Vorliegen von Magnesiumsulfat:

$$
\mathrm{MgSO}_{4}+\mathrm{Na}_{2} \mathrm{CO}_{3}+\mathrm{Ca}(\mathrm{OH})_{2}=\mathrm{CaCO}_{3}+\mathrm{Mg}(\mathrm{OH})_{2}+\mathrm{Na}_{2} \mathrm{SO}_{4} .
$$

\section{Die Ionenumtauschverfahren}

Nachdem wir bis jetzt Verfahren besprochen haben, die auf der Ausfällung von gelösten Verbindungen beruhen, gehen wir zu Methoden über, bei welchen feste Stoffe bestimmter Zusammensetzung, die sogenannten Austauscher, einzelne im Wasser enthaltene Ionen selektiv aufzunehmen vermögen. Beim Ionenumtauschverfahren lässt man das zu behandelnde Wasser durch ein Austauscherfilter perkolieren, wobei sich die 
chemischen Umsetzungen an der wirksamen Substanz praktisch momentan vollziehen, so dass ein Wasser der gewünschten Zusammensetzung abfliesst. Die Vorteile des Verfahrens bestehen darin, dass bei nur kurzer Kontaktzeit und weitgehend unabhängig von der Konzentration des Rohwassers ein vollständiger Umsatz erzielt werden kann und dass sich der erschöpfte Austauscher mit Hilfe von Chemikalien auf relativ einfache Weise wieder betriebsbereit machen lässt.

Strukturchemisch können die Austauscher als lockere Gerüste (Kristallgitter oder vernetzte Ketten) aufgefasst werden, welche in ihrem Netzwerk eine grosse Zahl ionogener, das heisst dissoziationsfähiger Gruppen enthalten. Diese können sein

bei den kationenaktiven Substanzen: $-\mathrm{COOH}$ oder $-\mathrm{SO}_{3} \mathrm{H}$, und bei den anionenaktiven Substanzen: $-\mathrm{NH}_{2}$ oder $-\mathrm{NR}_{3} \mathrm{OH}$.

Die Stoffe dissoziieren analog der sich frei in Lösung befindlichen Säuren, Basen oder Salze, das heisst, um das positiv oder negativ geladene Gerüst herum befinden sich die entgegengesetzt geladenen, frei beweglichen Gegenionen, zum Beispiel bei einem Kationenaustauscher - $\mathrm{COOH}-$ $-\mathrm{COO}^{-}+\mathrm{H}^{+}$oder bei einem Anionenaustauscher $-\mathrm{NH}_{3} \mathrm{OH} \longrightarrow-\mathrm{NH}_{3}{ }^{+}+$ $\mathrm{OH}^{-}$. Die beweglichen Gegenionen können durch andere Ionen ersetzt werden, beispielsweise $\mathrm{H}^{+}$durch $\mathrm{Na}^{+}, 2 \mathrm{H}^{+}$durch $\mathrm{Ca}^{++}, \mathrm{OH}^{-}$durch $\mathrm{Cl}^{-}$ oder $2 \mathrm{OH}^{-}$durch $\mathrm{SO}_{4}^{--}$.

Die modernen Kunstharzaustauscher, von welchen hier vor allem die Rede sein soll, werden durch Polykondensation oder durch Polymerisation geeigneter Ausgangssubstanzen hergestellt. Man erhält zum Beispiel aus meta-Phenylendiamin und Formaldehyd durch Polykondensation einen schwach basischen Anionenaustauscher oder aus Methakrylsäure und Divinylbenzol durch Polymerisation einen schwach sauren Kationenaustauscher.

Wird nun ein Austauscher mit einer ionisierten Lösung, zum Beispiel dem aufzubereitenden Wasser, in Berührung gebracht, so treten je nach der Art der anwesenden Stoffe die ionogenen Gruppen des Austauschers mit den Ionen der Lösung in Reaktion. Anders ausgedrückt: die Ionen des Wassers setzen sich an die Stellen der Gegenionen des Austauschers, wobei diese in die Lösung übertreten. Wegen des lockeren Aufbaus der Austauscher sind die im ganzen Gerüst verteilten ionogenen Gruppen für die Ionen in der zu perkolierenden Lösung zugänglich. Man nimmt an, dass das lockere Netzwerk nicht nur eine äussere Oberfläche, sondern auch eine sogenannte innere Oberfläche besitzt, zu welcher die Moleküle des Lö- 
sungsmittels - in unserem Falle das Wasser - und die in ihm gelösten Ionen gelangen können. Für diese Annahme spricht auch die grosse Quellbarkeit der Austauscher, zum Beispiel bei der Benetzung mit Wasser. Das Wasser wird vom Netzwerk adsorbiert, was die Quellung der Harze zur Folge hat. Das in den Austauscher eingedrungene Wasser bildet um die Harzpartikeln herum einen Flüssigkeitsfilm. Durch diesen Film dringen auch die Ionen der Aussenlösung infolge der Diffusion zu den ionogenen Gruppen ein, wo sich je nach den herrschenden Gleichgewichtsbedingungen die Austauschreaktionen abspielen.

Um sich einen Begriff von der Struktur und der Reaktionsfähigkeit der heute zur Verfügung stehenden Harzaustauscher machen zu können, seien noch folgende Zahlen erwähnt:

Die innere Oberfläche wird mit rund $500 \mathrm{~m}^{2} / \mathrm{g}$ Harz angegeben, eine sehr grosse Zahl im Vergleich zur eigentlichen Oberfläche von I g gekörntem Harz. Die Austauschkapazitäten variieren ungefähr zwischen 0,5 und Io Milliäquivalenten pro Gramm Substanz. Im Innern der Harzpartikel herrschen daher hohe Ionenkonzentrationen. Sie entsprechen Elektrolytlösungen mit Normalitäten von rund o,5-10.

Über die Selektivität sei ausgesagt, dass sie gemäss der Wertigkeitsregel von SCHulzE und HaRdY mit zunehmender Aufladung der beteiligten Ionen zunimmt. Demnach haftet zum Beispiel in der Reihe $\mathrm{Na}^{+}, \mathrm{Mg}^{++}$, $\mathrm{Al}^{+++}$das dreiwertige Aluminiumion am stärksten am Austauscher. Ferner steigt auch die Selektivität mit abnehmender Hydratation der Ionen gemäss den Reihen $\mathrm{Li}^{+}<\mathrm{Na}^{+}<\mathrm{K}^{+}<\mathrm{Rb}^{+}<\mathrm{Cs}^{+}$oder $\mathrm{F}^{-}<\mathrm{Cl}^{-}<\mathrm{Br}^{-}$ $<\mathrm{J}^{-}$, was in Übereinstimmung mit der Hofmeisterschen Reihe bei kolloidchemischen Vorgängen steht.

Die Berechnung der Ionenkonzentrationen, welche in der Aussenlösung und am Harz bei gegebenen Bedingungen vorliegen, wird teils auf Grund empirischer Formeln, teils mit Hilfe des Massenwirkungsgesetzes durchgeführt, wobei letzteres analog zu den Berechnungen bei Lösungen mit echt gelösten Ionen zu geschehen hat. Obgleich die rechnerische Ermittlung der Ionenverhältnisse bei Harzen aus verschiedenen Gründen noch fehlerhaft sein mögen, so lässt sich doch mit Hilfe der Formeln des Massenwirkungsgesetzes zeigen, dass zum Beispiel bei der Perkolation mit zunehmender Verdünnung der durchfliessenden Lösung der Anteil des höherwertigen Ions am Austauscher grösser wird. Umgekehrt folgt aus dieser Erkenntnis, dass bei der Regeneration, bei welcher meist ein höherwertiges Ion durch ein niederwertiges ersetzt werden muss, stark konzentrierte Lösungen angewendet werden müssen. 
Entbärtung durch Ionenumtausch. Die Austauscher werden vielfach zu Enthärtung von Leitungswasser verwendet. Hierzu wird in einer geeigneten Apparatur ein Kationenaustauscher eingefüllt und mit Kochsalzlösung behandelt, wobei das Harz mit Natriumionen aufgeladen wird. Nach dem Herausspülen des überschüssigen Kochsalzes lässt man das zu enthärtende Wasser durch den Austauscher hindurchfliessen. Dabei setzen sich die Kalzium- und Magnesiumionen an die Stellen, an welchen die Natriumionen waren. Das aus dem Enthärtungsapparat ausfliessende Wasser enthält nun nicht mehr die Kalzium- und Magnesiumsalze, sondern die entsprechenden Natriumsalze. Schematisch kann dies folgendermassen formuliert werden:

$$
\left.\left.\mathrm{Ca}\left(\mathrm{HCO}_{3}\right)_{2}+-\right) 2 \mathrm{Na}^{+}=-\right) \mathrm{Ca}^{++}+2 \mathrm{NaHCO}_{3} .
$$

(Das Zeichen -) bedeutet Kationenaustauscher, das Zeichen + ) Anionenaustauscher. -) $2 \mathrm{Na}^{+}$bedeutet, dass der Kationenaustauscher mit zwei Natriumionen beladen ist.)

Man erkennt somit, dass das enthärtete Wasser noch den gleichen Salzgehalt aufweist wie das Rohwasser. Es wurden lediglich die Kalzium- und Magnesiumionen durch die Natriumionen ersetzt. Der Austauscher kann nur eine bestimmte Menge von Kalzium- bzw. Magnesiumionen aufnehmen. Nach seiner Beladung muss er durch Behandlung mit Kochsalzlösung wieder gebrauchsfertig gemacht werden, was « regenerieren» genannt wird.

Entsalzung durcb Ionenaustausch. Verwendet man beim Regenerieren Salzsäure anstatt Kochsalzlösung, so entsteht ein mit Wasserstoffionen beladener Austauscher. Wird nun Leitungswasser durch diesen durchfliessen gelassen, so treten die Kalzium- und Magnesiumionen an die Stelle der Wasserstoffionen, das heisst, es entstehen die entsprechenden Säuren, zum Beispiel gemäss folgenden Gleichungen:

$$
\begin{gathered}
\left.\left.\mathrm{CaCl}_{2}+-\right) 2 \mathrm{H}^{+}=-\right) \mathrm{Ca}^{++}+2 \mathrm{HCl}, \\
\left.\left.\mathrm{Ca}\left(\mathrm{HCO}_{3}\right)_{2}+-\right) 2 \mathrm{H}^{+}=--\right) \mathrm{Ca}^{++}+2 \mathrm{H}_{2} \mathrm{CO}_{3} .
\end{gathered}
$$

Lässt man hierauf das Wasser noch durch einen Anionenaustauscher, der mit Hydroxylionen beladen ist, fliessen, so werden die Anionen gemäss folgender Gleichung ausgetauscht:

$$
\mathrm{H}_{2} \mathrm{CO}_{3}++12 \mathrm{OH}^{-}=+1 \mathrm{CO}_{3}^{--}+2 \mathrm{H}_{2} \mathrm{O} \text {. }
$$

Es ist somit durch die Kombination von Kationen- und Anionenaustauschern möglich, ein vollständig entsalztes Wasser herzustellen, das qualitativ einem destillierten Wasser entspricht oder es unter Umständen noch übertrifft. 


\section{Die Koagulationsverfahren}

Ein Oberflächenwasser, welches leicht trübe oder gefärbt ist oder sehr fein verteilte, nicht absetzbare Schwebestoffe enthält, lässt sich gewöhnlich weder durch Absetzen noch durch Filtration klären bzw. entfärben. Die verunreinigenden Stoffe sind teils kolloidal gelöste organische Verbindungen, teils feinste Suspensa organischer Natur oder mineralischer Herkunft (zum Beispiel Tone, Silikate). Zur Reinigung solcher Wässer dienen die Koagulationsverfahren, bei welchen einerseits bezweckt wird, den kolloidalen Zustand der Partikeln aufzuheben (Entstabilisierung), anderseits flockige Niederschläge erzeugt werden sollen, welche die Schmutzstoffe zu adsorbieren vermögen. Als Flockungsmittel dienen in der Regel Aluminiumsulfat $\left[\mathrm{Al}_{2}\left(\mathrm{SO}_{4}\right)_{3} \cdot 18 \mathrm{H}_{2} \mathrm{O}\right]$, Eisen(II)sulfat $\left(\mathrm{FeSO}_{4} \cdot 7 \mathrm{H}_{2} \mathrm{O}\right)$ und Eisen(III)chlorid $\left(\mathrm{FeCl}_{3} \cdot 6 \mathrm{H}_{2} \mathrm{O}\right)$. Die meisten Verunreinigungen in Oberflächenwässern sind negativ geladene Kolloide, die sich mit den positiven Metallhydroxyd-Kolloidpartikeln vereinigen und zu grösseren Flocken zusammenballen. Diese Reaktionen benötigen Zeit. In der Praxis wird so vorgegangen, dass zuerst das zu behandelnde Wasser mit den zugegebenen Reagenzien gut vermischt wird und hierauf der Mischung Zeit für die Flockenbildung gegeben wird.

\section{LITERATURVERZEICHNIS}

[t] T. E. Larson und A. M. Buswell, J. Amer. Wat. Wks. Ass. 34, I667 (I942).

[2] H. S. HARNEd und F. T. BonNer, J. Amer. chem. Soc. 67, 1026 (1945).

[3] H. S. Harned und R. S. Scholes jr., J. Amer. chem. Soc. 63, I706 (T94I).

[4] E. Nordeli, Water Treatment (Reinhold Publishing Corporation, New York 195I). 